This item was submitted to Loughborough's Research Repository by the author.

Items in Figshare are protected by copyright, with all rights reserved, unless otherwise indicated.

\title{
Open quantum systems, effective Hamiltonians, and device characterization
}

PLEASE CITE THE PUBLISHED VERSION

https://doi.org/10.1103/PhysRevB.96.134520

\section{PUBLISHER}

(C) American Physical Society

VERSION

VoR (Version of Record)

\section{PUBLISHER STATEMENT}

This work is made available according to the conditions of the Creative Commons Attribution-NonCommercialNoDerivatives 4.0 International (CC BY-NC-ND 4.0) licence. Full details of this licence are available at: https://creativecommons.org/licenses/by-nc-nd/4.0/

\section{LICENCE}

CC BY-NC-ND 4.0

\section{REPOSITORY RECORD}

Dwyer, Vincent, Stephen N.A. Duffus, and Mark Everitt. 2019. “Open Quantum Systems, Effective Hamiltonians, and Device Characterization". figshare. https://hdl.handle.net/2134/27339. 


\title{
Open quantum systems, effective Hamiltonians, and device characterization
}

\author{
S. N. A. Duffus, ${ }^{1,2}$ V. M. Dwyer, ${ }^{1,3, *}$ and M. J. Everitt ${ }^{1,2}$ \\ ${ }^{1}$ Quantum Systems Engineering Research Group, Loughborough University, Loughborough, Leicestershire LE11 3TU, United Kingdom \\ ${ }^{2}$ Department of Physics, Loughborough University, Leicestershire LE11 3TU, United Kingdom \\ ${ }^{3}$ Wolfson School of Mechanical, Electrical and Manufacturing Engineering, Loughborough University, \\ Leicestershire LE11 3TU, United Kingdom \\ (Received 11 July 2017; revised manuscript received 1 September 2017; published 27 October 2017)
}

\begin{abstract}
High fidelity models, which are able to both support accurate device characterization and correctly account for environmental effects, are crucial to the engineering of scalable quantum technologies. As it ensures positivity of the density matrix, one preferred model of open systems describes the dynamics with a master equation in Lindblad form. In practice, Linblad operators are rarely derived from first principles, and often a particular form of annihilator is assumed. This results in dynamical models that miss those additional terms which must generally be added for the master equation to assume the Lindblad form, together with the other concomitant terms that must be assimilated into an effective Hamiltonian to produce the correct free evolution. In first principles derivations, such additional terms are often canceled (or countered), frequently in a somewhat ad hoc manner, leading to a number of competing models. Whilst the implications of this paper are quite general, to illustrate the point we focus here on an example anharmonic system; specifically that of a superconducting quantum interference device (SQUID) coupled to an Ohmic bath. The resulting master equation implies that the environment has a significant impact on the system's energy; we discuss the prospect of keeping or canceling this impact and note that, for the SQUID, monitoring the magnetic susceptibility under control of the capacitive coupling strength and the externally applied flux results in experimentally measurable differences between a number of these models. In particular, one should be able to determine whether a squeezing term of the form $\hat{X} \hat{P}+\hat{P} \hat{X}$ should be present in the effective Hamiltonian or not. If model generation is not performed correctly, device characterization will be prone to systemic errors.
\end{abstract}

DOI: 10.1103/PhysRevB.96.134520

\section{INTRODUCTION}

For any complex system, if device characterization and design engineering are to be meaningful notions, it is necessary to have access to models that faithfully describe device behavior and performance when subjected to a wide range of environmental conditions. The ability to integrate billions of CMOS transistors onto a single chip relies on careful device characterization as well as knowledge of likely environmental conditions. A chip meant for liquid Helium applications will be characterized by lower junction capacitances, as a result of carrier freeze-out [1], whilst, if intended for space applications, it must be designed with radiation hardness in mind [2]. Successful quantum technologies will be no different in this regard.

In the quest to build a scalable quantum processor from Josephson-junction-based superconducting circuits, significant developments have been made recently, including enhanced coherence times [3], high fidelity state-preparation and measurement [4], and the demonstration of two-qubit gates [5]. To facilitate the fabrication of functional circuits, future qubits must be capable of long- and short-term storage, be frequency tunable, be capable of communication over both short and long ranges, and possess tunable couplings [6]. Decoherence presents a formidable obstacle to achieving this for superconducting qubits. As a result, the maintenance of a long coherence time has become a key problem in superconductor qubit research, making the need for a better

\footnotetext{
*v.m.dwyer@lboro.ac.uk
}

understanding of different types of environment a similarly key concern.

Until relatively recently, it had been assumed that the connection to ground of floating flux-qubits was poor, and that such qubits were immune to capacitive coupling. However, a recent study [6] has demonstrated the reactance to ground can become sizable, and that not protecting against this decoherence channel may explain some of the short coherence times obtained previously.

The equivalent design paradigm for a classical semiconductor (CMOS) computer allows a very detailed simulation of critical-path devices in a variety of environments (at fast/typical/slow mobility process corners, under mechanical stress, flicker or thermal noise, etc.) as well in a range of operational conditions (e.g., IR-drop on power lines) and including a number of reliability considerations (e.g., metal migration) before it is committed to silicon [7]. In the particular case of capacitive coupling, the contrast of the superconducting qubit to silicon CMOS is stark; indeed, CMOS is the chosen platform because of its excellent noise immunity. Understanding the impact of an environment is necessary for accurate characterization of a given quantum device, as devices which are not characterized under the same environmental conditions may not easily be compared, and a device characterized in a test environment may behave differently when in use.

\section{THE OPEN QUANTUM SYSTEM}

A common means of modeling open quantum systems is with reduced master equations [8-18], as these approximate 
the dynamics of the system of interest without the need to model the environment itself, and, because it guarantees certain nice properties (such as conservation of probability and complete positivity of the reduced (system) density matrix, and natural unravellings), it is usual to cast the master equation into Lindblad form [19], in which the Lindblad operators represent the action of the environment on the system. Exactly which operators to use usually involves a carefully constructed analysis of the system + environment, followed by a tracing out of environmental degrees of freedom. This process requires a number of approximations to be made, as well as some decisions about the form of the final equation. Most common is the Born-Markov (BM) approximation, which assumes a weak coupling between a quantum system and an environment, assumed to have no long-term memory. However, the BM approximation alone will not generally yield a quantum master equation of the required form. As a consequence, it is common to manipulate the resulting $\mathrm{BM}$ master equation by adding those terms necessary to complete the Lindblad structure, and by either absorbing any terms that present as free evolution into an effective system Hamiltonian, or by canceling them with heuristic counter terms.

The simplest example is that of quantum Brownian motion (QBM), with a harmonic potential, coupled to an Ohmic bath through the system's position operator $\hat{x}$, see, e.g., Ref. [9]. Here, a Lindblad master equation for the reduced density matrix $\hat{\rho}_{S}$ can be created from the Born-Markov development by (i) adding a term of the form $D_{\mathrm{pp}}\left[\hat{p},\left[\hat{p}, \hat{\rho}_{S}\right]\right]$, where $\hat{p}$ is the canonical conjugate momentum to $\hat{x}([\hat{x}, \hat{p}]=\mathrm{i} \hbar)$, and $D_{p p}$ is inversely proportional to the bath temperature $T$; and (ii) by moving terms proportional to $\left[\hat{x}^{2}, \hat{\rho}_{S}\right]$ (the Lamb shift term) and $\mu\left[\hat{x} \hat{p}+\hat{x} \hat{p}, \hat{\rho}_{S}\right]$ (a squeezing term, where $\mu$ depends on system-bath coupling strength) into an effective system Hamiltonian. The justification for the addition of the $D_{\mathrm{pp}}\left[\hat{p},\left[\hat{p}, \hat{\rho}_{S}\right]\right]$ term is often that, in the high $T$ limit, it is small and so amounts to a minimally invasive means of ensuring Lindblad form. The Lamb shift term $\left[\hat{x}^{2}, \hat{\rho}_{S}\right]$ is generally canceled by the inclusion of an identical (counter) term in either the system or interaction Hamiltonian on the grounds that it otherwise constitutes an unphysical frequency renormalization. With these changes, the QBM master equation takes on Lindblad form, with a single, generalized annihilator Lindblad, $\hat{b}=c_{1} \hat{x}+c_{2} \hat{p}$ [9].

Up to this point, what has been described is the standard high-temperature (Lindblad) version of the Caldiera-Leggett equation [20], where it is also argued that, for many systems, a counter term arises to cancel the Lamb shift in a truly detailed analysis of microscopic dynamics. The final (squeezing term) $\mu[\hat{x} \hat{p}+\hat{p} \hat{x}, \hat{\rho}]$, has been discussed in the literature on a number of occasions. On one hand, its presence (uncountered) in an effective Hamiltonian is necessary to ensure translational invariance [21], that Erhenfest's theorem is satisfied [19,21-24], and a correct quantum to classical transition [25-29].

On the other hand, the inclusion of a counterterm $-\lambda[\hat{x} \hat{p}+$ $\hat{p} \hat{x}, \hat{\rho}]$ in the system Hamiltonian has occasionally been part of an ansatz, which assumes the most general second order Lindblad master equation possible, using a single (generalized annihilator) Lindblad, first order in system variables $\hat{x}$ and $\hat{p}$, together with a general second order Hamiltonian. Its properties have been investigated as a function of $\lambda$. Setting $\lambda=\mu$ cancels the squeezing term completely, while $\lambda \neq 0$ (providing a partial cancellation) gives additional flexibility to add desirable properties to the master equation, such as reasonable low $T$ behavior [21,24]. This does, however, sacrifice translational invariance [19] and Ehrenfest [23].

The problems in the derivation of the Lindblads, appropriate to a particular system-environment interaction, are clearer still when considering a particle in an anharmonic potential, such as is the case for a superconducting quantum interference device (SQUID). Here, the heuristic adding/canceling of terms becomes much more involved [30], which necessarily raises difficult issues as high-precision control of such a system inevitably requires precise device characterization [31]. The purpose of this paper is to highlight such problems, using the SQUID as an example system, and to investigate whether a measurement of its magnetic susceptibility might be capable of providing some resolution of these difficulties.

\section{AN EXAMPLE ANHARMONIC POTENTIAL-THE SQUID}

It is worth stressing, to begin, that the problems encountered in the present case will inevitably occur, to a greater or lesser extent, for any anharmonic potential $V(\hat{x})$, and our choice here is largely for the purposes of definitiveness. We consider the following standard model of a SQUID, with charge $\hat{Q}$ and flux $\hat{\Phi}$ as canonically conjugate variables (so that $[\hat{\Phi}, \hat{Q}]=$ $\mathrm{i} \hbar$ ), coupled both inductively and capacitively to an Ohmic bath environment, modeled as an infinite set of harmonic oscillators, mode index $n$, with charge $\hat{Q}_{n}$ and flux $\hat{\Phi}_{n}$ $\left(\left[\hat{\Phi}_{n}, \hat{Q}_{m}\right]=\mathrm{i} \hbar \delta_{n m}\right)$ [30]. The dimensionless total Hamiltonian $\hat{\mathcal{H}} / \hbar \omega_{0}$ may be written as a sum of the Hamiltonians of the SQUID $\left(\hat{\mathcal{H}}_{S}\right)$, the bath $\left(\hat{\mathcal{H}}_{B}\right)$, and the coupling between them $\left(\hat{\mathcal{H}}_{I}\right)$ :

$$
\begin{aligned}
\hat{\mathcal{H}}_{S} & =\frac{\hat{X}^{2}}{2}+\frac{\hat{P}^{2}}{2}-\frac{v}{\omega_{0}} \cos \left(\sqrt{\frac{\beta v}{\omega_{0}}} \hat{X}+\frac{2 \pi \Phi_{x}}{\Phi_{0}}\right)+\hat{\mathcal{H}}_{L S} \\
& =\hat{\mathcal{H}}_{0}+\hat{\mathcal{H}}_{L S}, \\
\hat{\mathcal{H}}_{B} & =\sum_{n} \frac{\hat{X}_{n}^{2}}{2}+\frac{\hat{P}_{n}^{2}}{2}, \\
\hat{\mathcal{H}}_{I} & =-\sum_{n} \kappa_{n}\left(\hat{X} \hat{X}_{n}+g \hat{P} \hat{P}_{n}\right)=-\hat{X} \hat{B}_{X}-g \hat{P} \hat{B}_{P},
\end{aligned}
$$

where $L$ and $C$ are the system's inductance and capacitance, $\omega_{0}=1 / \sqrt{L C}$, and we have introduced the dimensionless operators $\hat{P}=\hat{Q}\left(\hbar C \omega_{0}\right)^{-1 / 2}, \hat{P}_{n}=\hat{Q}_{n}\left(\hbar C_{n} \omega_{0}\right)^{-1 / 2}$, $\hat{X}=\hat{\Phi}\left(\hbar L \omega_{0}\right)^{-1 / 2}, \quad \hat{X}_{n}=\hat{\Phi}_{n}\left(\hbar L_{n} \omega_{0}\right)^{-1 / 2}, \quad$ and $\beta v / \omega_{0}=$ $4 \pi^{2} \hbar / \Phi_{0}^{2} C$, while $\Phi_{0}$ denotes the flux quantum. Here, $\hat{B}_{X}$ and $\hat{B}_{P}$ are shorthand versions of the bath functions. The externally applied flux, $\Phi_{x}$, controls the phase of the cosine in Eqs. (1), which describe the coupling across the Josephson junction (energy $=\hbar v)$ [32]. The strengths of the environmental couplings are determined by coupling constants $\kappa_{n}$ and by $g$, the ratio of inductive to capacitive coupling. $\hat{\mathcal{H}}_{L S}$ is a Lamb shift Hamiltonian, which is included generally to cancel unwanted frequency shift terms that are generated in the Lindbald process 
[20]. However, it is useful to leave it unspecified at this stage.

We assume, as is common, that the initial state of the System + Bath is separable and the bath is so large that it rapidly relaxes back to its initial thermal equilibrium state so that we may take $\hat{\rho}_{B}$ as effectively independent of time and $\hat{\rho}(t)=$ $\hat{\rho}_{S}(t) \otimes \hat{\rho}_{B}$. Similarly, we assume, for simplicity, that the bath is described by an Ohmic spectral function of the form

$$
J(\omega)=\frac{2 \gamma \omega \Omega^{2}}{\pi\left(\Omega^{2}+\omega^{2}\right)},
$$

with damping rate $\gamma$ and bath cut-off frequency $\Omega$.

In the Schrödinger picture, the BM master equation may be expressed as [9]

$$
\frac{\mathrm{d} \hat{\rho}_{S}(t)}{\mathrm{d} t}=\mathcal{L}\left[\hat{\rho}_{S}\right]+\mathcal{K}\left[\hat{\rho}_{S}\right]=-\mathrm{i}\left[\hat{\mathcal{H}}_{S}, \hat{\rho}_{S}(t)\right]-\int_{0}^{\infty} \mathrm{d} \tau \operatorname{Tr}_{B}\left[\hat{\mathcal{H}}_{I},\left[\hat{\mathcal{H}}_{I}(-\tau), \hat{\rho}_{S}(t) \otimes \hat{\rho}_{B}\right]\right]
$$

The first term, $\mathcal{L}\left[\hat{\rho}_{S}\right]$, describes free von Neumann evolution of the system and the second, the dissipator $\mathcal{K}\left[\hat{\rho}_{S}\right]$, describes nonunitary loss.

Substituting $\hat{\mathcal{H}}_{I}$ from Eqs. (1) into the dissipator integral, and evaluating the bath correlation functions (i.e., $\left\langle\hat{B}_{X} \hat{B}_{X}(-\tau)\right\rangle$, etc.), yields $[9,30]$

$$
\begin{aligned}
\mathcal{K}[\hat{\rho}]= & \frac{\gamma \Omega}{2 \omega_{0}} \int_{0}^{\infty} \mathrm{d} \tau e^{-\Omega \tau}\left(\frac{2 \Omega}{\omega_{0}}\left(\mathrm{i}\left[\hat{X},\left\{\hat{X}(-\tau), \hat{\rho}_{S}\right\}\right]+\mathrm{i} g^{2}\left[\hat{P},\left\{\hat{P}(-\tau), \hat{\rho}_{S}\right\}\right]+g\left[\hat{X},\left[\hat{P}(-\tau), \hat{\rho}_{S}\right]\right]-g\left[\hat{P},\left[\hat{X}(-\tau), \hat{\rho}_{S}\right]\right]\right)\right. \\
& \left.-\left(\left[\hat{X},\left[\hat{X}(-\tau), \hat{\rho}_{S}\right]\right]+g^{2}\left[\hat{P},\left[\hat{P}(-\tau), \hat{\rho}_{S}\right]\right]-\mathrm{i} g\left[\hat{X},\left\{\hat{P}(-\tau), \hat{\rho}_{S}\right\}\right]+\mathrm{i} g\left[\hat{P},\left\{\hat{X}(-\tau), \hat{\rho}_{S}\right\}\right]\right)\right),
\end{aligned}
$$

where $\{\cdot, \cdot\}$ denotes anticommutation.

Expanding the correlation-time dependent observables $\hat{X}(-\tau)$ and $\hat{P}(-\tau)$ to first order in $\tau$ using a BCH series [9]

$$
\begin{aligned}
& \hat{X}(-\tau)=\hat{X}-\mathrm{i} \tau\left[\mathcal{H}_{S}, \hat{X}\right]+\mathcal{O}\left(\tau^{2}, \gamma\right) \approx \hat{X}-\tau \hat{P}, \\
& \hat{P}(-\tau)=\hat{P}-\mathrm{i} \tau\left[\mathcal{H}_{S}, \hat{P}\right]+\mathcal{O}\left(\tau^{2}, \gamma\right) \approx \hat{P}+\tau\left(\hat{X}+\frac{v}{\omega_{0}} \sqrt{\frac{\beta v}{\omega_{0}}} \sin \left(\sqrt{\frac{\beta v}{\omega_{0}}} \hat{X}+\frac{2 \pi \Phi_{x}}{\Phi_{0}}\right)\right),
\end{aligned}
$$

effectively corresponds, after time integration, to an expansion in $\omega_{0} / \Omega$, and thus to a broadband bath (large $\Omega$ ). The presence of $\gamma$ in the error terms in Eqs. (5) arises from the Lamb shift Hamiltonian $\mathcal{H}_{L S}$, i.e., from counter terms included to offset unphysical frequency shifts $[33,34]$. Here, such terms are assumed to be in the system, rather than the interaction Hamiltonian, as this keeps the theoretical development of Eq. (3) somewhat simpler. Finally, evaluating the resulting integrals yields the following Born-Markov master equation:

$$
\begin{aligned}
\frac{\mathrm{d} \hat{\rho}_{S}}{\mathrm{~d} t^{\prime}}= & -\mathrm{i}\left[\hat{\mathcal{H}}_{S}, \hat{\rho}_{S}\right]-\frac{\mathrm{i} \gamma}{\omega_{0}}\left(1+g^{2}-g\right)\left[\hat{X},\left\{\hat{P}, \hat{\rho}_{S}\right\}\right]-\frac{\gamma}{\omega_{0}}\left(g+\frac{1}{2}\right)\left[\hat{X},\left[\hat{X}, \hat{\rho}_{S}\right]\right]+\frac{\gamma}{2 \Omega}\left(1-g^{2}\right)\left[\hat{X},\left[\hat{P}, \hat{\rho}_{S}\right]\right] \\
& +\mathrm{i} \frac{2 \gamma \Omega}{\omega_{0}^{2}}\left[\frac{\hat{X}^{2}}{2}, \hat{\rho}\right]+\mathrm{i} \frac{2 \gamma g \Omega}{\omega_{0}^{2}}\left[\frac{\hat{P}^{2}}{2}, \hat{\rho}\right]-\frac{\mathrm{i} \gamma}{\omega_{0}} g\left(g-\frac{1}{2}\right)\left[\{\hat{X}, \hat{P}\}, \hat{\rho}_{S}\right]-\frac{\gamma}{\omega_{0}} g\left(1+\frac{g}{2}\right)\left[\hat{P},\left[\hat{P}, \hat{\rho}_{S}\right]\right] \\
& +\frac{\mathrm{i} \gamma g}{\omega_{0}} \sqrt{\frac{\beta v}{\omega_{0}}}\left[\frac{\omega_{0}}{2 \Omega} \hat{X}+g \hat{P},\left\{\sin \left(\sqrt{\frac{\beta \omega_{0}}{v}} \hat{X}+2 \pi \frac{\Phi_{x}}{\Phi_{0}}\right), \hat{\rho} S\right\}\right]-\frac{\gamma g}{\omega_{0}} \sqrt{\frac{\beta v}{\omega_{0}}}\left[\hat{X}+\frac{g \omega_{0}}{2 \Omega} \hat{P},\left[\sin \left(\sqrt{\frac{\beta \omega_{0}}{v}} \hat{X}+2 \pi \frac{\Phi_{x}}{\Phi_{0}}\right), \hat{\rho} \hat{\rho}_{S}\right]\right] .
\end{aligned}
$$

In the case that the coupling is purely inductive $(g=0)$, only the first five terms on the right hand side of Eq. (6) survive, so that the external flux control parameter $\Phi_{x}$ disappears from the dissipator at first order, appearing only in the second order $\mathrm{BCH}$ model in that case [30].

As Eq. (6) is a nonrotating wave (NRW) equation, to assume Lindblad form [19], both of the following adjustments are required: some terms that present in the form of a unitary evolution must be combined with $\mathcal{H}_{S}$ to produce an effective Hamiltonian and some new terms must be added to the dissipator [24,35]. At the end of this process, Eq. (6) may be written in the Lindblad form

$$
\frac{\mathrm{d} \hat{\rho}}{\mathrm{d} t}=-\mathrm{i}\left[\hat{\mathcal{H}}_{\mathrm{eff}}, \hat{\rho}\right]+\frac{1}{2} \sum_{j}\left\{\left[\hat{L}_{j}, \hat{\rho} \hat{L}_{j}^{\dagger}\right]+\left[\hat{L}_{j} \hat{\rho}, \hat{L}_{j}^{\dagger}\right]\right\}
$$

where $\mathcal{H}_{\text {eff }}$ is the effective Hamiltonian and the $\hat{L}_{j}$ are Lindblad operators. We consider each of these adjustments now in detail.

\section{THE EFFECTIVE HAMILTONIAN}

Of particular interest is the question of what one might do with the terms left over from the process of ensuring a Lindblad 
form for master equation. As in the case of the QBM with a harmonic potential, all such terms present in the form of a unitary evolution (i.e., as $\mathrm{i}\left[\hat{A}, \hat{\rho}_{S}\right]$ for some Hermitian operator $\hat{A})$, which may then be included in an effective Hamiltonian. However, there appears to be no clear means of assessing whether such terms should be kept or canceled (countered)either partially or completely. Retaining all terms for now, we obtain an effective Hamiltonian $\hat{\mathcal{H}}_{\text {eff }}=\hat{\mathcal{H}}_{0}+\hat{\mathcal{H}}_{X P}+\hat{\mathcal{H}}_{P S}+$ $\hat{\mathcal{H}}_{X S}+\hat{\mathcal{H}}_{L S}-\hat{\mathcal{H}}_{\delta}$, where

$$
\begin{aligned}
\hat{\mathcal{H}}_{X P}= & \frac{\gamma}{2 \omega_{0}}\left(\frac{3 g^{2}}{2}-g+\frac{1}{2}\right)\{\hat{X}, \hat{P}\}, \\
\hat{\mathcal{H}}_{X S}= & -\frac{\gamma g}{4 \Omega} \sqrt{\frac{\beta v}{\omega_{0}}}\{\hat{X}, \hat{S}\}, \quad \hat{\mathcal{H}}_{P S}=-\frac{\gamma g^{2}}{2 \omega_{0}} \sqrt{\frac{\beta \nu}{\omega_{0}}}\{\hat{P}, \hat{S}\}, \\
\hat{\mathcal{H}}_{\delta}= & \frac{2 \gamma \Omega}{\omega_{0}^{2}}\left(\frac{\hat{X}^{2}}{2}+g \frac{\hat{P}^{2}}{2}\right) \\
& +\frac{\gamma g^{2} \beta}{4 \Omega} \cos \left(\sqrt{\frac{\beta \omega_{0}}{v}} \hat{X}+2 \pi \frac{\Phi_{x}}{\Phi_{0}}\right)
\end{aligned}
$$

where we have introduced the symbol $S$ as a shorthand for the sine terms in Eq. (6). Note that the final term $\hat{\mathcal{H}}_{\delta}$ causes a renormalization of all terms in the system Hamiltonian $\hat{\mathcal{H}}_{S}$. The first two cause the anticipated "frequency" shift of the $L C$ oscillator, while the last term renormalizes the tunneling energy of the Josephson junction. In other words, the environment appears to affect all terms in the system Hamiltonian, raising the question: What in $\hat{\mathcal{H}}_{\text {eff }}$ should one cancel with a carefully chosen $\hat{\mathcal{H}}_{L S}$ as being unphysical? For the SQUID, this is an open question, the answer to which one might seek from rehearsing standard QBM analysis. In the case of a harmonic potential, the sine $(\hat{S})$ and cosine terms are missing from Eqs. (8), giving an effective Hamiltonian $\hat{\mathcal{H}}_{\text {eff }}=\hat{\mathcal{H}}_{0}+\hat{\mathcal{H}}_{X P}+\hat{\mathcal{H}}_{L S}-\hat{\mathcal{H}}_{\delta}$, where

$$
\begin{aligned}
& \hat{\mathcal{H}}_{X P}=\frac{\gamma}{2 \omega_{0}}\left(\frac{3 g^{2}}{2}-g+\frac{1}{2}\right)\{\hat{X}, \hat{P}\}, \\
& \hat{\mathcal{H}}_{\delta}=\frac{2 \gamma \Omega}{\omega_{0}^{2}}\left(\frac{\hat{X}^{2}}{2}+g \frac{\hat{P}^{2}}{2}\right) .
\end{aligned}
$$

$\hat{\mathcal{H}}_{\delta}$ is routinely canceled by introducing an identical counterterm, i.e., by setting $\hat{\mathcal{H}}_{L S}=\hat{\mathcal{H}}_{\delta}$, while the impact of $\hat{\mathcal{H}}_{X P}$ has previously been discussed in terms of frequency shifts and squeezing [30,32], and in other contexts too [19,21,21-26,29]; however, it has also been the source of some debate as discussed in the Introduction: arranging for a counterterm in $\hat{\mathcal{H}}_{L S}$ allows the master equation to display additional properties [21], while such cancellation does come at the cost of sacrificing translational invariance [19] and Ehrenfest [23].

Returning to the present case of the SQUID, and as a extension of QBM, we also choose to set $\hat{\mathcal{H}}_{\delta}=\hat{\mathcal{H}}_{L S}$ in the remainder of the analysis. The additional cosine term in Eqs. (8) is sufficiently small as to not impact on the conclusions of this section (for all intents and purposes, Fig. 1 and Fig. 2 remain the same), whether it is canceled or not. What then remains is the question of what to do with the anticommutator terms; generally the most significant of which is the squeezing $\hat{X} \hat{P}+\hat{P} \hat{X}$ term. Before proceeding, it is worth making the
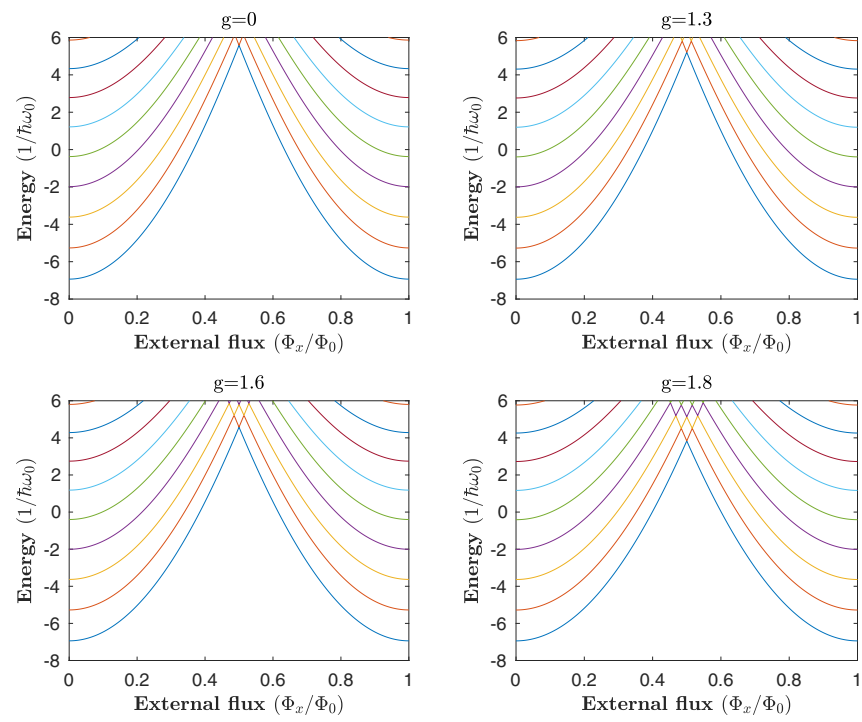

FIG. 1. The energy eigenvalues of the effective system Hamiltonian $\hat{\mathcal{H}}_{\text {eff }}$, including all anticommutator contributions from the environment, as a function of external flux $\Phi_{x}$, for various coupling ratios $g$. Results are obtained with parameter values of Josephson energy $\hbar v=6.693 \times 10^{-22} \mathrm{~J}=7.74 \hbar \omega_{0}$, capacitance $C=5 \times$ $10^{-15} \mathrm{~F}$, inductance $L=3 \times 10^{-10} \mathrm{H}$, damping rate $\gamma=0.05 \omega_{0}$, and bath cut-off frequency $\Omega=10 \omega_{0}$. Increasing the coupling ratio has a noticeable effect on the energy level structure.

observation that, for a general potential $V(\hat{X}), V^{\prime}(\hat{X})-\hat{X}$ would replace $\sqrt{\beta v / \omega_{0}} \hat{S}$ in Eqs. (8), and the cosine term in $\hat{\mathcal{H}}_{\delta}$ would be replaced by a term proportional to $V^{\prime \prime}(\hat{X})$.

The impact of the anticommutator terms on the lowest five energy eigenvalues of $\hat{\mathcal{H}}_{\text {eff }}$, as a function of the external flux $\Phi_{x}$, may be seen in Fig. 1, for a variety of values of $g$. Here,

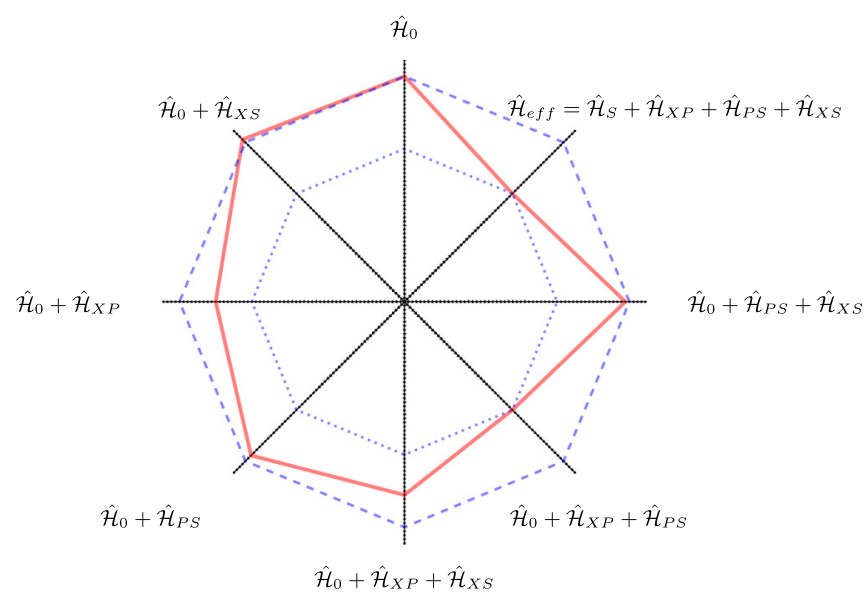

FIG. 2. Spiderweb Diagram. The solid line shows the lowest eigenvalue for the SQUID Hamiltonian plus various combinations of anticommutator terms that arise from coupling to the environment, as labeled. Dashed and dotted lines show, for comparison, the lowest eigenvalues of $\hat{\mathcal{H}}_{0}$ (the isolated SQUID) and $\hat{\mathcal{H}}_{\text {eff }}$ (corrected for environmental contributions, but with $\hat{\mathcal{H}}_{L S}=\hat{\mathcal{H}}_{\delta}$ ), respectively. Note that the contributions of $\hat{\mathcal{H}}_{X P}, \hat{\mathcal{H}}_{X S}$, and $\hat{\mathcal{H}}_{P S}$ are not independent, and that the combination $\hat{\mathcal{H}}_{X P}+\hat{\mathcal{H}}_{X S}$ accounts for the majority of the alteration. The origin corresponds to $E=0$, the outer radius to $E=6.0 \hbar \omega_{0}$. Here, $\Phi_{x}=0.5 \Phi_{0}, g=1.8$. 
the SQUID parameters are chosen to be $L=3 \times 10^{-10} \mathrm{H}$, $C=5 \times 10^{-15} \mathrm{~F}$, giving an $L C$ frequency $\omega_{0}=8.16 \times 10^{11}$ rads $^{-1}$. In dimensionless units, we assume a weak coupling of $\gamma / \omega_{0}=0.05$, a broad spectrum Ohmic bath with cutoff $\Omega=10 \omega_{0}$ and a Josephson coupling energy of $\nu / \omega_{0}=7.74$, in this case corresponding to a critical current $I_{C} \approx 3 \mu \mathrm{A}$. All calculations were preformed in the Fock basis of the harmonic part of the system Hamiltonian, truncated at 140 basis states to ensure accuracy of the numerics. Even at levels significantly below this value, results are insensitive to where the truncation is applied.

Figure 2 shows the contribution to the lowest energy level of the anticommutator terms, $\hat{\mathcal{H}}_{X P}, \hat{\mathcal{H}}_{P S}$, and $\hat{\mathcal{H}}_{X P}$, in Eqs. (8), both individually and in pairs, to illustrate how the cancellation, or not, of such terms will alter the system's ground state. It is clear from both of these figures that these effects are non-negligible, and that any form of ad hoc process to deal with their contribution is likely to lead to erroneous system models. That one of the terms is already known to be important in the quantum to classical transition [25-29] implies that there are physically important implications here that almost certainly should not be ignored.

The magnetic susceptibility for a SQUID ring [36],

$$
\chi_{0}\left(\Phi_{x} ; g\right)=-L \frac{\partial^{2} E_{0}\left(\Phi_{x} ; g\right)}{\partial \Phi_{x}^{2}},
$$

provides a useful mechanism to probe the dependence of the lowest energy eigenvalue on the external flux $\Phi_{x}$, and the coupling ratio $g$. Plotted as $\chi_{0}\left(\Phi_{x} ; g\right) / L$ in Fig. 3 , it shows significant variations with both $\Phi_{x}$ and $g$.

An important conclusion of the analysis to this point is that, by arranging an adjustable capacitive coupling and varying the external flux, a measurement of $\chi_{0}$ can provide an empirical test of the presence of the anticommutator terms and, hence, whether they are indeed physical and should be kept, or are merely an artifact of the process needed to shoehorn the BornMarkov master equation into Lindblad form and need to be canceled.

We end this section by noting that the interpretation of this experiment (the variation of the susceptibility with $g$, and $\Phi_{x}$ shown in Fig. 3) depends upon an acceptance that it is valid, at least to a reasonable level of approximation, to describe the decoherence seen in the case of a SQUID by a Markovian Lindblad master equation. From any given initial state, short

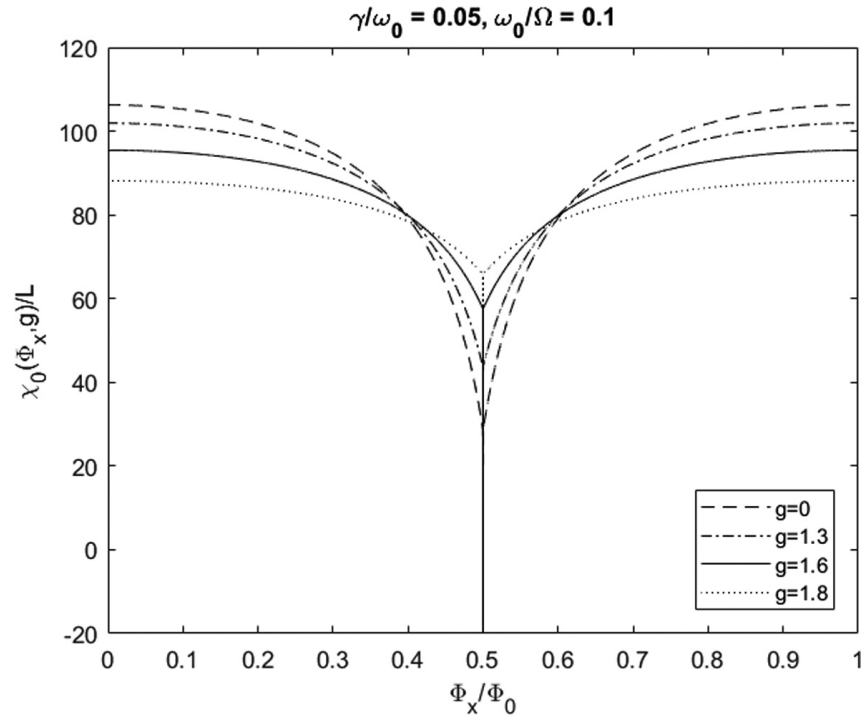

FIG. 3. Ground state magnetic susceptibility as a function of the external flux $\Phi_{x}$, and capacitive coupling strength $g$, with all anticommutator terms included in $\hat{\mathcal{H}}_{\text {eff }}$. As the coupling strength is increased, the external flux dependence of the magnetic susceptibility flattens out, showing measurable differences that could form the basis of experiments to certify specific candidate open-system models.

time evolution is likely to be non-Markovian; however, for times longer than the relaxation time of the system, evolution is expected to be approximately Markovian [37]. However, such an experiment may also provide evidence in support, or otherwise, of the use of the Markovian approximation in low temperature systems such as this.

\section{OBTAINING THE LINDBLADS}

The terms that must be added to a BM master equation, such as Eq. (6), are generally clear; in this case the missing term is $\left[\hat{S},\left[\hat{S}, \hat{\rho}_{S}\right]\right]$. In the case of a harmonic potential, the missing term is of the form $\left[\hat{P},\left[\hat{P}, \hat{\rho}_{S}\right]\right]$, with its coefficient chosen to ensure one makes the smallest change possible to the master equation. This is sometimes described as minimal invasion [9]. Here, it is clear from Eq. (6) that the Lindblads will be formed from linear combinations of the components $\hat{X}, \hat{P}$, and $\hat{S}$, whose weights may be obtained by diagonalizing the coefficient matrix [9]

$$
a_{A B}=\frac{\gamma}{\omega_{0}}\left(\begin{array}{ccc}
2 g+1 & -\mathrm{i}\left(1+g^{2}-g\right)-\xi\left(1-g^{2}\right) & g \sqrt{\frac{\beta \nu}{\omega_{0}}}(1+\mathrm{i} \xi) \\
\mathrm{i}\left(1+g^{2}-g\right)-\xi\left(1-g^{2}\right) & 2 g+g^{2} & g^{2} \sqrt{\frac{\beta \nu}{\omega_{0}}}(\xi+\mathrm{i}) \\
g \sqrt{\frac{\beta \nu}{\omega_{0}}}(1-\mathrm{i} \xi) & g^{2} \sqrt{\frac{\beta \nu}{\omega_{0}}}(\xi-\mathrm{i}) & 0
\end{array}\right)
$$

for $A, B \in\{X, P, S\}$, defined (with $\xi=\omega_{0} / 2 \Omega$ ) such that $a_{A B}$ is the coefficient of $\left[\hat{A},\left[\hat{B}, \hat{\rho}_{S}\right]\right]$ in Eq. (6). As is often the case with nonrotating-wave Born-Markov equations, the coefficient matrix is not positive semidefinite, and so does not conserve probability. To ensure physicality, we proceed in what might be considered to be the standard manner and alter $a_{\mathrm{SS}}$ just sufficient to make the coefficient matrix positive semidefinite and its eigenvalues non-negative. Effectively, this is accomplished in a minimally invasive [9] manner by fixing $\operatorname{det}(a)=0$, i.e., by altering $a_{\mathrm{SS}}$ to

$$
a_{S S}=\frac{\beta \gamma \nu}{\omega_{0}^{2}} \frac{4 g^{4}+8 \xi^{2} g^{3}}{\left(-g^{4}+2 g^{2}-1\right)\left(1+\xi^{2}\right)+4 g\left(g^{2}+1\right)},
$$




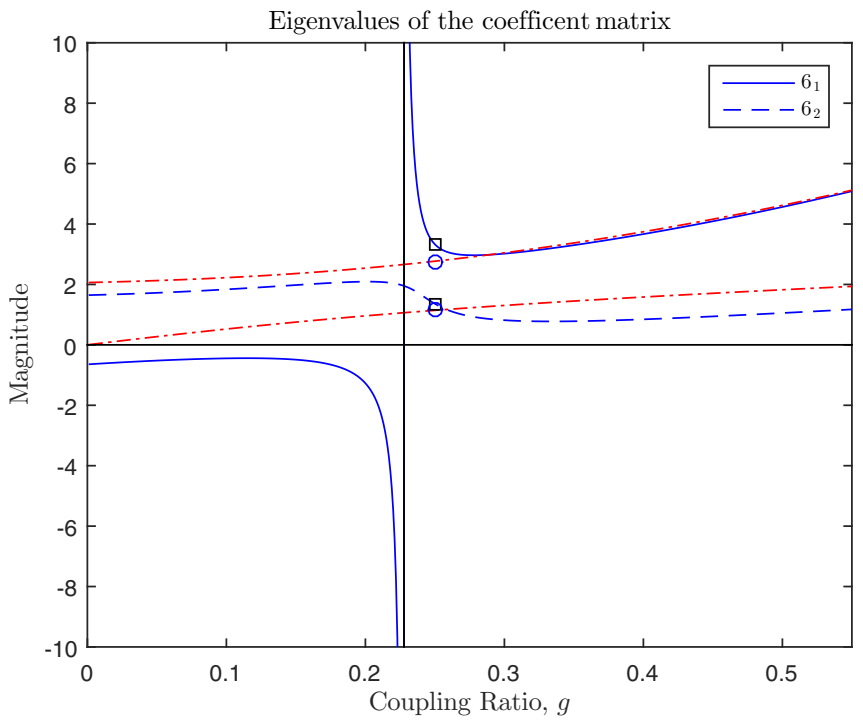

FIG. 4. The two nonzero eigenvalues of the coefficent matrix $a_{A B}$ [Eq. (11) ], which determine the strength of the Lindblad operators, as a function of coupling ratio $g$. Making the minimum addition to ensure Lindblad form constrains $g$, for $\xi=0.25$, as here, $g \in(0.227,4.4)$. An alternative, more invasive change to $a_{A B}$, which affects both $a_{\mathrm{PP}}$ and $a_{\mathrm{SS}}$, produces the dot-dashed eigenvalues. Markers indicate values at $g=0.25$, used in decoherence calculations.

together with the constraint that $0.23 \lesssim g \lesssim 4.4$, whose exact bounds are weakly dependent on $\xi \leqslant 0.25$. This procedure ensures the two remaining eigenvalues are positive. The range of $g$ values may be extended, but only at the cost of a more significant change to the matrix $a$; this necessarily increases $\operatorname{det}(a)$ away from 0 and also, as a consequence, introduces a third Lindblad.

The origin of the lower limit on $g$ is clear from a consideration of the case of purely inductive coupling [30], when the coefficient matrix reduces to

$$
a_{A B}=\frac{\gamma}{\omega_{0}}\left(\begin{array}{cc}
1 & -\mathrm{i}-\xi \\
\mathrm{i}-\xi & 0
\end{array}\right)
$$

in the basis $\{\hat{X}, \hat{P}\}$. Now guided by the same notions, and to ensure positive eigenvalues, we take $a_{\mathrm{PP}}=\gamma\left(1+\xi^{2}\right) / \omega_{0}$. This choice sets $\operatorname{det}(a)=0$, and leads to a generalized annihilator Lindblad of the form $c_{1} \hat{X}+c_{2} \hat{P}$. Essentially, this is the same process as the adding of $D_{\mathrm{pp}}\left[\hat{p},\left[\hat{p}, \hat{\rho}_{S}\right]\right]$ in the case of Brownian motion in a harmonic potential, as discussed in the Introduction. It is clear that if the coefficient matrix in Eq. (11) is to lead to positive eigenvalues, then, roughly speaking, $\omega_{0} a_{\mathrm{PP}} / \gamma:=2 g+g^{2}$ must be larger than $1+\xi^{2}$, and thus $g$ must be bounded below.

It is possible to use an alternative method, which removes the lower bound on $g$, but involves adding terms to Eq. (6) in two stages. First, one completes the Lindblad form for the inductive channel only (i.e., $g=0$ ) by adjusting $a_{\mathrm{PP}}$, and then opens the capacitive channel $(g \neq 0)$ and completes the process again, adjusting $a_{\mathrm{SS}}$; for midrange values of $g$ there is little difference between the results obtained (see the dot-dashed lines in Fig. 4); the dominant Lindblad is unchanged beyond $g \approx 0.25$, though the less dominant

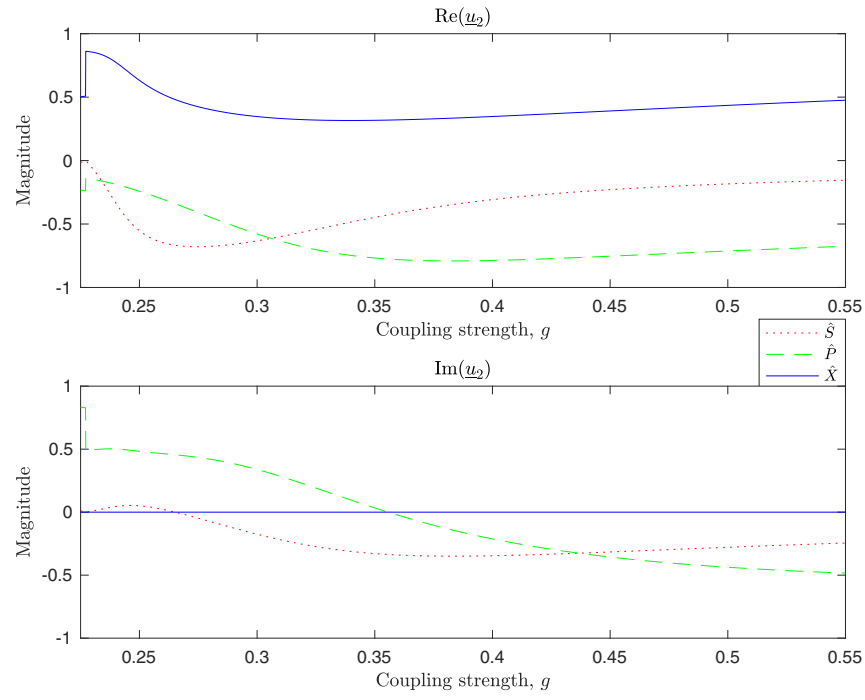

FIG. 5. The real and imaginary coefficients of the component operators of the dominant Lindblad, eigenvalue $\lambda_{1}$, as a function of coupling ratio $g$. In addition to the expected generalized annihilator contributions, there are also significant contributions from $\hat{S}$. This shows that the contribution from the Josephson junction term to the Lindblads are significant, and depend nontrivially on the coupling strength $g$. It is also noteworthy that this naturally leads to an external flux dependence in the Lindblad.

Lindblad is somewhat stronger in this second method. Either way, the upper limit on $g$ remains. However one chooses to proceed, it is clear that using this minimal invasion, as a guiding principle, is rather ad hoc and does not provide a continuous, or a particularly satisfactory means of introducing extra decoherence channels and that some other method is necessary. As our main conclusions are largely independent of the particular choice, for our present purposes we choose $a_{\mathrm{SS}}$ as given in Eq. (12) and accept the restriction on $g$.

With this adjustment to $a_{\mathrm{SS}}$, diagonalizing the coefficient matrix in Eq. (11) leads to real, positive eigenvalues $\lambda_{j}$, and associated eigenvectors $\underline{u}_{j}^{\mathrm{T}}$ in the $(\hat{X}, \hat{P}, \hat{S})$ basis. The Lindblad operators are then created as $\hat{L}_{j}=\sqrt{\lambda_{j}}(\hat{X}, \hat{P}, \hat{S}) \underline{u}_{j}^{\mathrm{T}}$. As $\operatorname{det}(a)=0$, one of the eigenvalues is zero for all $g$, meaning that the associated Lindblad may be neglected. For midrange $g$ values, the two remaining eigenvalues $\lambda_{j}$, displayed in Fig. 4, possess a slowly increasing dependence on the strength of capacitive coupling. The weighting of the basis terms $\hat{X}, \hat{P}$, and $\hat{S}$ in each Lindblad is presented in Figs. 5 and 6. These figures show, respectively, the real and imaginary contribution of each operator to each Lindblad. In addition to an anticipated generalized annihilator contributions of the form $\hat{b}=c_{1} \hat{X}+$ $c_{2} \hat{P}$, there are now also significant contributions arising from the Josephson term $\hat{S}$ and, consequently, a significant role in decoherence for the external flux $\Phi_{x}$. We consider this impact in the next section.

\section{MODELING DECOHERENCE}

With Lindblads obtained for a particular coupling strength, it is possible to examine the impact of the environment on the SQUID through Eq. (7). In the range $0.25 \lesssim g \lesssim 4.4$, 


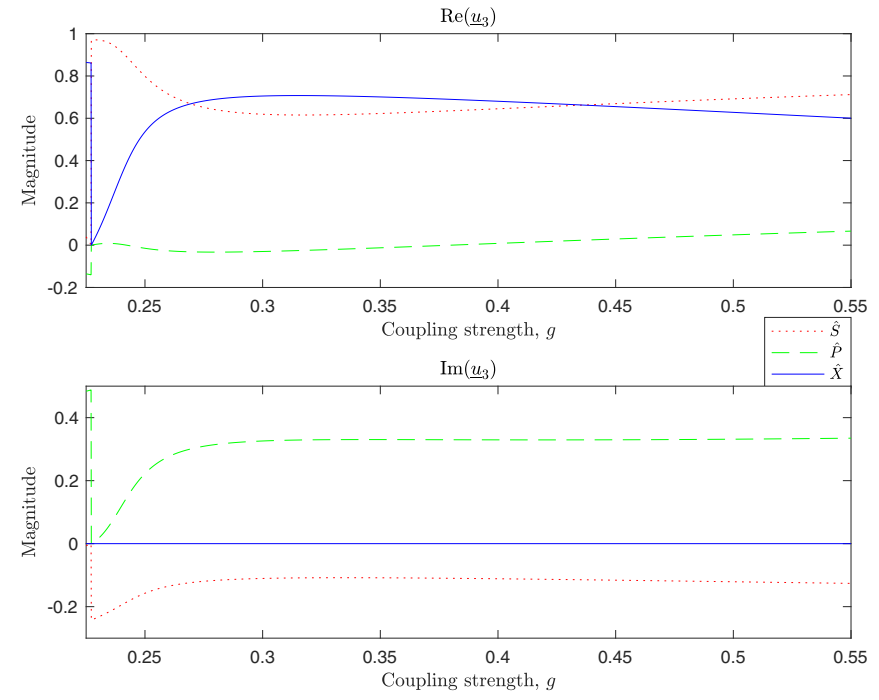

FIG. 6. As in Fig. 5 for the second Lindblad, eigenvalue $\lambda_{2}$.

we obtain very similar Lindblads from the two methods used in the previous section so, to complete the analysis we investigate the decoherence, assuming all anti-commutator terms are included, with $g=0.25$. Here we take the purity of the steady state $P_{\infty}=\operatorname{Tr}\left[\rho(t \rightarrow \infty)^{2}\right]$, and its dependence on the externally applied magnetic flux $\Phi_{x}$, as a measure of the effect of environmental degrees of freedom. This dependence is shown in Fig. 7, for a number of coupling ratios, with a bath cutoff frequency of $\Omega=2 \omega_{0}$. The Lindblads responsible for these dissipative effects may be drawn from Figs. 5 and 6 , and the steady state purity is obtained from solving Eq. (7) with the left-hand side set to zero. The dashed curve in Fig. 7 , for example, corresponds to the case of $g=0.25$ when the

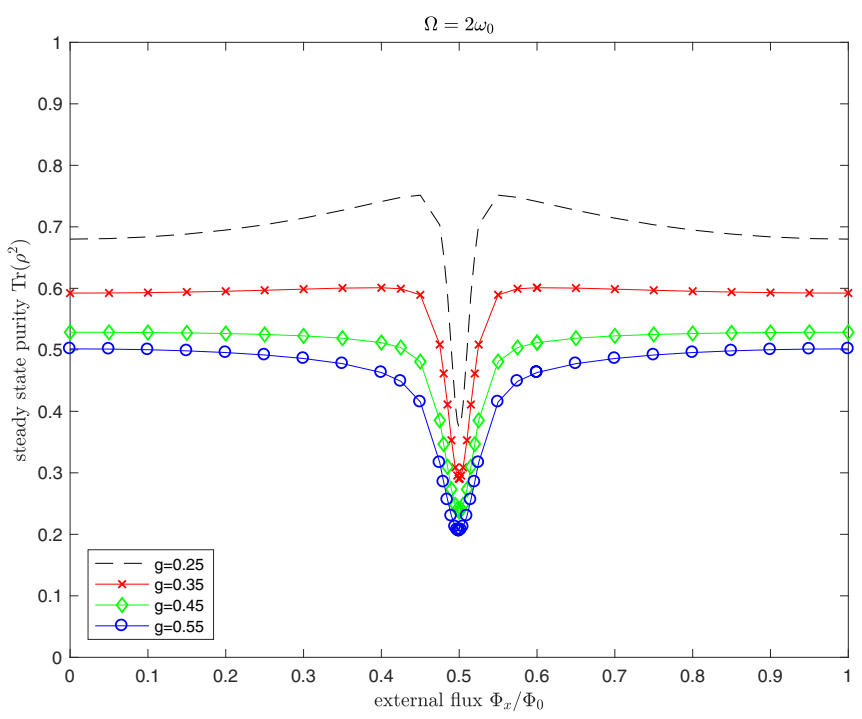

FIG. 7. The steady state purity of a SQUID coupled inductively and capacitively to an Ohmic bath as a function of external flux for various capacitive coupling strengths at a bath cut-off frequency of $\Omega=2 \omega_{0}$. Note that the well created about $\Phi_{x}=0.5 \Phi_{0}$, and flattens and widens as capacitive coupling strength is increased.

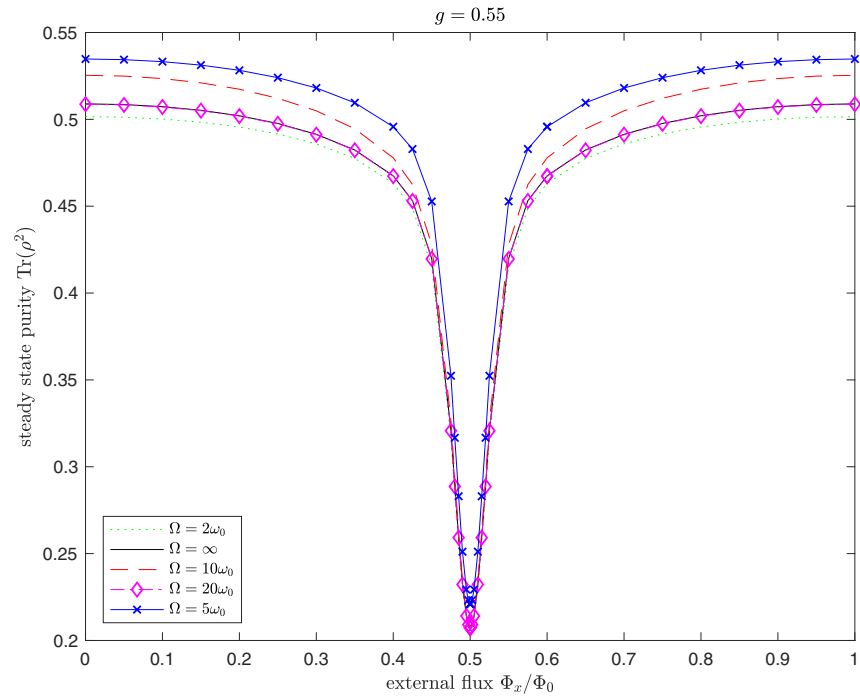

FIG. 8. The steady state purity of a SQUID coupled inductively and capacitively to an Ohmic bath as a function of external flux for various bath cutoff frequencies at a coupling ratio of $g=0.55$. A drop in purity below a value of a half suggests leakage to higher energy levels than simply the ground state.

Lindbalds $L_{1}$ and $L_{2}$ are given by

$$
\begin{aligned}
& \frac{L_{1}}{\sqrt{1.36 \gamma / \omega_{0}}}=0.63 \hat{X}+(0.48 \mathrm{i}-0.24) \hat{P}+(0.05 \mathrm{i}-0.55) \hat{S}, \\
& \frac{L_{2}}{\sqrt{3.33 \gamma / \omega_{0}}}=0.54 \hat{X}+(0.22 \mathrm{i}-0.015) \hat{P}+(0.8-0.16 \mathrm{i}) \hat{S}
\end{aligned}
$$

(see markers in Fig. 4).

Figure 7 indicates increased mixing with increasing capacitive coupling. At low $g$ the shape of the purity curve has a strong resemblance to that found when the decoherence is due to an annihilation operator [30], while at higher $g$ values, the well at the half integer flux broadens. The impact on the steady state obtained through increasing the coupling strength to $g=0.55$ is also significant, and occurs in a manner that is relatively insensitive to the bath cutoff beyond $\Omega \geqslant 10-20 \omega_{0}$, as shown in Fig. 8. Noticeably, the steady state purity in both Figs. 7 and 8 falls below 0.5 , and an analysis of the density matrix $\hat{\rho}_{S}$ indicates that a number of low excited states are involved in the mixture. This behavior is different from the spontaneous decay found in flux qubits caused by $1 / f$ noise, where the system would be expected to reach a purity $\operatorname{Tr}\left[\hat{\rho}^{2}\right]$ of approximately 0.5 [38].

\section{CONCLUSIONS}

Making new quantum technologies scalable will rely on the ability to perform careful modeling and simulation. Any such simulation framework will require high-fidelity models and accurate characterization methods, to allow precision control and enable the necessary error correction. However, it seems clear that, beyond the simplest case of a high temperature harmonic potential, deriving Lindblad master equations from the Born-Markov approximation becomes rather ad hoc, as the decision to include (as being physically real) or to exclude 
(through the use of counterterms) is not supported well enough by our knowledge of the systems involved. Even in that simplest case (QBM), there is still some disagreement about whether a squeezing term of the form $\hat{X} \hat{P}+\hat{P} \hat{X}$ should be kept or canceled [21-24].

For Lindblad decoherence of an open system with an anharmonic potential $V(\hat{X})$, a microscopic analysis such as Born-Markov is necessary to determine the form of the Lindblads. We show here that, in addition to frequency shifts (which are routinely canceled) and a squeezing $\hat{X} \hat{P}+\hat{P} \hat{X}$ term (which is generally kept, but occasionally partly or wholly canceled), forcing the master equation into Lindblad form generates a number of other, similar terms. In the case of the SQUID, all terms in the free Hamiltonian $H_{0}$ are affected by the environment, including a renormalization of the Josephson junction tunneling energy, which one must then choose to counter or not.

In addition to the squeezing term, which here is the dominant contribution of the remaining environmental impact, pairwise terms of the form $H_{A B}=c_{A B}\{A, B\}$ arise for all pairs $A, B \in\left\{\hat{X}, \hat{P}, V^{\prime}(\hat{X})\right\}$, which too must be considered for inclusion or countering in the same way. Thus, in principle, for a general potential $V(\hat{X})$, we must make decisions on whether to cancel (or adjust) each of the $\delta_{k}, 1 \leqslant k \leqslant 6$, in the effective Hamiltonian

$$
\begin{aligned}
\mathcal{H}_{\mathrm{eff}}= & \frac{\hat{P}^{2}}{2}+V(\hat{X})+\delta_{1} \frac{\hat{X}^{2}}{2}+\delta_{2} \frac{\hat{P}^{2}}{2}+\delta_{3} V^{\prime \prime}(\hat{X}), \\
& \delta_{4}\{\hat{X}, \hat{P}\}+\delta_{5}\left\{P, V^{\prime}(\hat{X})\right\}+\delta_{6}\left\{\hat{X}, V^{\prime}(\hat{X})\right\}
\end{aligned}
$$

Careful measurements are needed to justify the steps in (what might reasonably be termed) the standard method that we have used. However, the SQUID system is useful in this regard, as it has simple and adjustable control parameters (the external flux $\Phi_{x}$ and the relative strength $g$ of capacitive coupling), which together appear to provide sufficient means to interrogate the energy level structure through measurement of the magnetic susceptibility, and thus the effective Hamiltonian. As we have shown, the additional anticommutator terms will make a measurable difference and, experimentally, the ability to create superconducting devices coupled to an artificially constructed bath of harmonic oscillators is well within the state-of-the-art. Hence, it will be possible to experimentally verify whether, for example, the squeezing term $\hat{X} \hat{P}+\hat{P} \hat{X}$ should be canceled by a counterterm or not.
[1] N. Yoshikawa, T. Tomida, M. Tokuda, Q. Liu, X. Meng, S. R. Whiteley, and T. V. Duzer, IEEE Trans. Appl. Supercond. 15, 267 (2005).

[2] G. Pouiklis, G. Kottaras, A. Psomoulis, and E. Sarris, Int. J. Electron. 100, 913 (2013).

[3] A. A. Houck, J. A. Schreier, B. R. Johnson, J. M. Chow, J. Koch, J. M. Gambetta, D. I. Schuster, L. Frunzio, M. H. Devoret, S. M. Girvin, and R. J. Schoelkopf, Phys. Rev. Lett. 101, 080502 (2008).

[4] E. Jeffrey, D. Sank, J. Y. Mutus, T. C. White, J. Kelly, R. Barends, Y. Chen, Z. Chen, B. Chiaro, A. Dunsworth, A. Megrant, P. J. J. O’Malley, C. Neill, P. Roushan, A. Vainsencher, J. Wenner, A. N. Cleland, and J. M. Martinis, Phys. Rev. Lett. 112, 190504 (2014).

[5] J. Plantenberg, P. de Groot, C. Harmans, and J. Mooij, Nature 447, 836 (2007).

[6] M. Steffen, F. Brito, D. DiVincenzo, S. Kumar, and M. Ketchen, New J. Phys. 11, 033030 (2009).

[7] T. H. Morshed et al., BSIM4v4.7 MOSFET Model-User's Manual, University of California, Berkley (2011).

[8] E. Arrigoni, M. Knap, and W. von der Linden, Phys. Rev. Lett. 110, 086403 (2013).

[9] H. Breuer and F. Petruccione, The Theory of Open Quantum Systems (OUP, Oxford, UK, 2007).

[10] J. C. Dyre, Phys. Rev. Lett. 58, 792 (1987).

[11] V. Giovannetti and G. M. Palma, Phys. Rev. Lett. 108, 040401 (2012).

[12] L.-A. Wu, G. Kurizki, and P. Brumer, Phys. Rev. Lett. 102, 080405 (2009).

[13] L. Ferialdi, Phys. Rev. Lett. 116, 120402 (2016).

[14] M. J. W. Hall, J. D. Cresser, L. Li, and E. Andersson, Phys. Rev. A 89, 042120 (2014).
[15] D. Maldonado-Mundo, P. Öhberg, B. W. Lovett, and E. Andersson, Phys. Rev. A 86, 042107 (2012).

[16] C. Joshi, P. Öhberg, J. D. Cresser, and E. Andersson, Phys. Rev. A 90, 063815 (2014).

[17] B. Vacchini, Phys. Rev. Lett. 117, 230401 (2016).

[18] L. Diósi and L. Ferialdi, Phys. Rev. Lett. 113, 200403 (2014).

[19] G. Lindblad, Rep. Math. Phys. 10, 393 (1976).

[20] A. Caldeira and A. Leggett, Ann. Phys. 149, 374 (1983).

[21] S. Gao, Phys. Rev. Lett. 79, 3101 (1997).

[22] A. Sndulescu and H. Scutaru, Ann. Phys. 173, 277 (1987).

[23] H. M. Wiseman and W. J. Munro, Phys. Rev. Lett. 80, 5702 (1998).

[24] A. Lampo, S. H. Lim, J. Wehr, P. Massignan, and M. Lewenstein, Phys. Rev. A 94, 042123 (2016).

[25] R. Schack, T. A. Brun, and I. C. Percival, J. Phys. A: Math. Gen. 28, 5401 (1995).

[26] M. J. Everitt, New J. Phys. 11, 013014 (2009).

[27] M. Everitt, W. Munro, and T. Spiller, Phys. Lett. A 374, 2809 (2010).

[28] M. J. Everitt, W. J. Munro, and T. P. Spiller, J. Phys: Conference Series 306, 012045 (2011).

[29] M. J. Everitt, W. J. Munro, and T. P. Spiller, Phys. Rev. A 79, 032328 (2009).

[30] S. N. A. Duffus, K. N. Bjergstrom, V. M. Dwyer, J. H. Samson, T. P. Spiller, A. M. Zagoskin, W. J. Munro, K. Nemoto, and M. J. Everitt, Phys. Rev. B 94, 064518 (2016).

[31] J. H. Cole, S. G. Schirmer, A. D. Greentree, C. J. Wellard, D. K. L. Oi, and L. C. L. Hollenberg, Phys. Rev. A 71, 062312 (2005).

[32] S. J. Devitt, W. J. Munro, and K. Nemoto, Rep. Prog. Phys. 76, 076001 (2013).

[33] J. Diggins, J. F. Ralph, T. P. Spiller, T. D. Clark, H. Prance, and R. J. Prance, Phys. Rev. E 49, 1854 (1994). 
[34] T. P. Spiller, D. A. Poulton, T. D. Clark, R. J. Prance, and H. Prance, Int. J. Mod. Phys. B 5, 1437 (1991); L. Vinet and A. Zhedanov, J. Phys. A: Math. Theor. 44, 085201 (2011).

[35] W. J. Munro and C. W. Gardiner, Phys. Rev. A 53, 2633 (1996).

[36] R. J. Prance, J. E. Mutton, E. P. Shephard, T. D. Clark, H. Prance, and T. P. Spiller, SQUID '85, Superconducting Quantum Interference Devices and their Applications, Proceedings of the
Third International Conference on Superconducting Quantum Devices, Berlin (West), 25-28 June, 1985, Vol. 3, edited by H. D. Hahlbohm and H. Lubbig (Walter de Gruyter, Berlin, 1985).

[37] B. Gardas, J. Luczka, A. Ptok, and J. Dajka, PLoS ONE 10, e0143912 (2015).

[38] F. Yoshihara, K. Harrabi, A. O. Niskanen, Y. Nakamura, and J. S. Tsai, Phys. Rev. Lett. 97, 167001 (2006). 